\title{
Analysis of fly fishing rod casting dynamics
}

\author{
Gang Wang ${ }^{\mathrm{a}, *}$ and Norman Wereley ${ }^{\mathrm{b}}$ \\ ${ }^{a}$ Department of Mechanical and Aerospace Engineering, University of Alabama in Huntsville, Huntsville, AL, USA \\ ${ }^{\mathrm{b}}$ Department of Aerospace Engineering, University of Maryland, College Park, MD, USA
}

Received 10 April 2009

Revised 22 August 2010

\begin{abstract}
An analysis of fly fishing rod casting dynamics was developed comprising of a nonlinear finite element representation of the composite fly rod and a lumped parameter model for the fly line. A nonlinear finite element model was used to analyze the transient response of the fly rod, in which fly rod responses were simulated for a forward casting stroke. The lumped parameter method was used to discretize the fly line system. Fly line motions were simulated during a cast based on fly rod tip response, which was used as the initial boundary condition for the fly line. Fly line loop generation, propagation, and line turn-over were simulated numerically. Flexible rod results were compared to the rigid rod case, in which the fly tip path was prescribed by a given fly rod butt input. Our numerical results strongly suggest that nonlinear flexibility effects on the fly rod must be included in order to accurately simulate casting dynamics and associated fly line motion.
\end{abstract}

\section{Introduction}

The literature of fishing is the richest among all sports, and its history dates back to 2000 B.C. [1,2]. Even for the subset of fly fishing, much literature is available. However, a significant fraction of fly fishing literature is devoted to its history, rod makers, casting techniques, fishing experiences, etc. There is a lack of literature about the technology of fly rods in terms of technical rod analysis, rod design, and rod performance evaluation. This motivated Phillips [2] to write "The Technology of Fly Rods," which is an in-depth examination of the design of the modern fly rod, its history and its role in fly fishing. He reviewed modern composite fly rod design, evaluation, and casting of the line and fly, in engineering terms. In his discussion of the fly rod design process, Phillips speculated that fly rod design/manufacture would be achieved using computer-aided approaches in the future. However, this represents a challenge for the fly rod industry, because current fly rod design and manufacture are often based on experience derived from a history empirical fabrication and test, and little information is publicly accessible about these proprietary rod design methodologies. To address this issue, we developed a comprehensive fly rod analysis of casting dynamics consisting primarily of a nonlinear finite element model to analyze a fly rod based on its geometric and material properties, and a lumped parameter fishing line model.

The modern high performance fly rod is composed mainly of graphite-epoxy composite materials. Fly rods have evolved from natural wood materials such as bamboo, metallic materials, to fiber-glass, and graphite-epoxy composites [2]. Our focus is on those fly rods that are fabricated using graphite-epoxy composite materials. A fly rod is composed of 2, 3, or 4 sections, and each section is typically a tapered hollow tube with a circular cross section. Each section can be assembled by incorporating ferrules [2]. In our study, a 3-piece fly rod with sleeve ferrules was considered.

Spolek [3] developed an early analysis of fly casting dynamics. The fly line was represented by three segments. These were the upper traveling line, the front loop, and the lower stationary line. This representation captured the

\footnotetext{
* Corresponding author: Professor of Aerospace Engineering, Associate Fellow AIAA, Member AHS, Fellow ASME, IOP. Tel.: 301405 1927; Fax: 301314 9001; E-mail: wereley@umd.edu.
} 
basic physics of fly casting. For each segment, the geometry of the line was prescribed. The traveling and stationary line were modeled as straight and parallel to the horizontal direction and the front loop had a semi-circular shape. The work and energy balance method was used to calculate the time history of the fly line for each segment. Finally, the attached fly velocity time history during a cast was obtained. Different taper geometry in the fly line affected the final fly velocity. A refined drag model was given by Lingrad [4] to account for the aerodynamics of a fly line. Robson [5] relaxed Spolek's assumption and presented a more detailed model for the fly line during forward casting. The lumped parameter method was used to discretize the fly line motions. The fly line was modeled as many small rigid filaments, each having only one rotational degree of freedom, and each filament was connected to its neighbor by massless hinges. The butt(or handle) rotation angle was prescribed and validated using data extracted from videos of human casting motions. The fly rod was first assumed to be a rigid body, so that tip position, velocity, and acceleration were the kinematic boundary conditions for the fly line model. A numerical scheme was developed to solve for the fly line motion during a cast. Bending of the rod was accounted for by introducing a modified rod tip path. The significant contribution of this work was that the numerical simulations of fly line motion during forward casting were performed and compared to actual casting results. The agreement was quite reasonable. The above research laid the foundation for the analysis of fly casting dynamics via simulation studies.

Recently, Gatti [6] developed a new continuum model of fly line dynamics using cable elements and a numerical algorithm for simulating fly casting was presented. The results of fly line deformation during casting were presented in Gatti and Perkins [7]. Gatti [6] also considered the fly rod/line system during the casting. The motion of the fly rod during casting was modeled as the superposition of a rigid rotation of the whole rod and a linear deformation about the rod's current configuration. The fly rod/line coupled and uncoupled models finally yielded similar results. Experimental casting was conducted to validate the analysis using a 'yarnrod' to emulate a fly rod. They concluded that fly rod tip path was the key to analyzing fly line motion during casting because it provided the boundary condition.

Ohnishi and Matsuzaki [8] applied a commercial nonlinear finite element package to analyze the large deformation of a graphite composite fishing rod due to a tip loading. A nonlinear finite element package, ADINA(Automatic Dynamic Incremental Nonlinear Analysis), was used [9]. Experiments were conducted to validate the finite element predictions of both the deflection and strains in the fishing rod due to a tip force. This was the first time that finite element method was applied to the fishing rod analysis. Hendry and Hubbard [10] studied fly rod casting simulations using a dynamic finite element model based on commercial ABAQUS package. Measured fly rod tip path data [11] were used to validate the analysis. However, application of such a highly sophisticated 3D finite element package as ADINA, to the fishing rod, which is only a simple beam like structure, obfuscates insights that might be gleaned from a simple 1D analysis. Therefore, a simple and accurate nonlinear finite element method for fly rod analysis is needed.

Because most modern fly rods are fabricated by the graphite-epoxy composite materials, the geometrically-exact composite beam models can be used to capture the dynamic behavior of fish rods [12,13]. In addition, the fly rods are hollow and tapered beam structures. The corresponding thin-walled geometrically-exact composite beam model [14] and tapered beam model [15] are available as well. A particular case was proposed by Simo and Vu-Quoc [16], in which an inertial frame was used to study the dynamics of flexible isotropic beam under large overall motions. In this model, the inertial frame was selected to describe the system kinetic and potential energies of a beam [17, 18]. The kinetic energy is then in a quadratic form and uncoupled. But the potential energy is nonlinear, where the finite strain theory was used. The finite strain beam theories were studied by Simo and Vu-Quoc [16], Reissner [19], and Antman [20], For a plane beam case, the strain expressions were given by Reissner [19]. In his model, less simplification and linearization were involved, and the strain was exactly determined in the deformed configuration.

In this study, we develop an analysis of fly fishing rod casting dynamics. The finite strain beam theory (or geometrically-exact beam model) $[16,19,20]$ was adopted to determine transient responses of a fly rod. We then used Robson's lumped parameter approach [5] to calculate fly line motion during a cast. Finally the fly fishing rod nonlinear deformation and fly line motion are simulated during a cast.

A 3-piece graphite-epoxy composite experimental fly rod was developed and used in our study. Experiments were conducted to validate our predictions of the load-deflection curve due to a tip force. Fly rod transient response was calculated under a forward casting stroke. The fly line was modeled using the lumped parameter method proposed by Robson [5]. Finally, we simulated fly rod casting dynamics and fly line loop generation, propagation, and line 


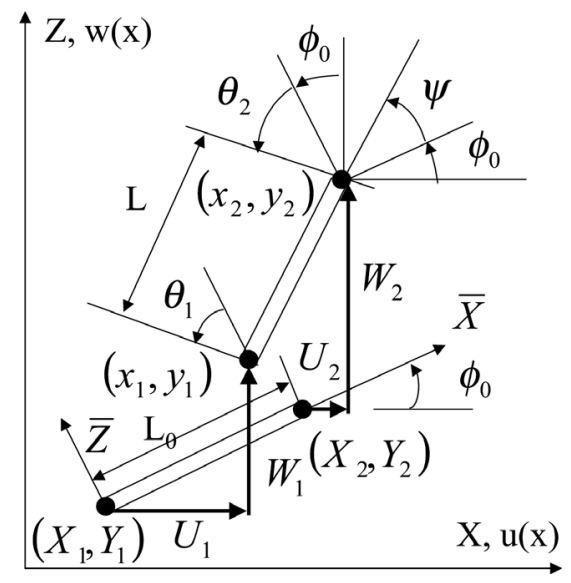

Fig. 1. Two-nodes nonlinear finite element based on finite strain beam theory.

turn-over were represented. The results were compared to results of a rigid fly rod case. In both rigid and flexible rod cases, we used identical fly rod butt motion to those used by Robson [5]. The nonlinear flexibility effects on the fly fishing rod must be included in order to accurately capture casting dynamics and associate fly line motion. In the following sections, we will present these in detail.

\section{Fly rod nonlinear finite element model}

Reissner [19] developed a finite strain beam theory for plane beam problems. Simo and Vu-Quoc [16] further exploited this approach and applied it to the dynamics of a flexible beam under large motions, which is similar to the case of fly rod deformation during a cast. All variables were defined with respect to the fixed frame. The key idea is to express the nonlinear strain of the deformed configuration in terms of unknown displacements, which are defined with respect to the fixed coordinates. The Newton-Raphson method [21] was used to iteratively solve for the displacement in the nonlinear finite element model. In the finite strain beam theory, we included the shearing deformation, which leads to the Timosenko beam theory where rotation angle is the independent variable and does not equal the slope of transverse displacement. By doing this, we obtain a simple kinematic relationship between strain and displacements. As discussed in [16,19,22,23], the nonlinear beam axial strain, $\epsilon$, shear strain, $\gamma$, and bending curvature, $\kappa$, can be expressed in terms of axial displacement $u(x)$, transverse displacement, $w(x)$, and rotation, displacement, $\theta$, as follows for a straight beam:

$$
\begin{aligned}
\epsilon & =\left(1+\frac{d u}{d x}\right) \cos \theta+\frac{d w}{d x} \sin \theta-1 \\
\gamma & =\frac{d u}{d x} \cos \theta-\left(1+\frac{d u}{d x}\right) \sin \theta \\
\kappa & =\frac{d \theta}{d x}
\end{aligned}
$$

The strain expressions are the same as those obtained by Antman [20], Simo and Vu-Quoc [16], and Vu-Quoc and Ebcioglu [22], in which the strains were derived in a vector form. The next step is to apply the finite element techniques to discretize the beam system. Figure 1 shows a two-node geometrically nonlinear finite element based on the finite strain beam theory, which has been known as a geometrically nonlinear Timosenko beam element. The element is not aligned to the $X$ axis for general consideration, which has an initial angle $\phi_{0}$. The nodal degrees of freedom were defined in the fixed frame except that the rotation angle $\theta_{1}$ and $\theta_{2}$ were calculated with respect to the initial element orientation. All displacements were linearly interpolated within an element using nodal degrees of freedom. 


$$
\begin{aligned}
u(x) & =N_{1}(x) U_{1}+N_{2}(x) U_{2} \\
w(x) & =N_{1}(x) W_{1}+N_{2}(x) W_{2} \\
\theta(x) & =N_{1}(x) \theta_{1}+N_{2}(x) \theta_{2}
\end{aligned}
$$

where the interpolation functions, $N_{1}(x)$ and $N_{2}(x)$ were defined as

$$
\begin{aligned}
& N_{1}(x)=1-\frac{x}{L} \\
& N_{2}(x)=\frac{x}{L}
\end{aligned}
$$

Before we derive the strain and displacement relations, we first demonstrate some geometric relationships as shown in Fig. 1. Given the node coordinates at node $1\left(X_{1}, Y_{1}\right)$, and node $2\left(X_{2}, Y_{2}\right)$, the initial reference angle $\phi_{0}$ is determined by

$$
\begin{aligned}
\cos \phi_{0} & =\frac{X_{2}-X_{1}}{L_{0}} \\
\sin \phi_{0} & =\frac{Y_{2}-Y_{1}}{L_{0}}
\end{aligned}
$$

where the element original length $L_{0}$ is given by

$$
L_{0}=\sqrt{\left(X_{2}-X_{1}\right)^{2}+\left(Y_{2}-Y_{1}\right)^{2}}
$$

The orientation of the deformed beam segment is described by the angle $\phi_{0}+\psi$ and it can be expressed by:

$$
\begin{aligned}
& \cos \left(\phi_{0}+\psi\right)=\frac{x_{2}-x_{1}}{L} \\
& \sin \left(\phi_{0}+\psi\right)=\frac{y_{2}-y_{1}}{L}
\end{aligned}
$$

where

$$
\begin{aligned}
x_{1} & =X_{1}+U_{1} \\
x_{2} & =X_{2}+U_{2} \\
y_{1} & =Y_{1}+W_{1} \\
y_{2} & =Y_{2}+W_{2} \\
L & =\sqrt{\left(x_{2}-x_{1}\right)^{2}+\left(y_{2}-y_{1}\right)^{2}}
\end{aligned}
$$

Solving the trigonometric relations in Eqs (3) and (5) for $\psi$, yields

$$
\begin{aligned}
& \cos \psi=\frac{\left(X_{2}-X_{1}\right)\left(x_{2}-x_{1}\right)+\left(Y_{2}-Y_{1}\right)\left(y_{2}-y_{1}\right)}{L L_{0}} \\
& \sin \psi=\frac{\left(X_{2}-X_{1}\right)\left(y_{2}-y_{1}\right)-\left(Y_{2}-Y_{1}\right)\left(x_{2}-x_{1}\right)}{L L_{0}}
\end{aligned}
$$

In order to derive the elemental stiffness matrices, we need to obtain the strain and displacement variational relationship,

$$
\delta h=B \delta q
$$

where $\delta$ is the variational operator. Here, $\mathbf{h}$ is the strain vector and all strains were given in Eq. 1 , and $q$ is the nodal displacement vector, given by

$$
\begin{aligned}
h & =\left[\begin{array}{lll}
\epsilon & \gamma & \kappa
\end{array}\right]^{T} \\
q & =\left[\begin{array}{llllll}
U_{1} & W_{1} & \theta_{1} & U_{2} & W_{2} & \theta_{2}
\end{array}\right]^{T}
\end{aligned}
$$


The $B$ matrix is calculated by taking partial derivatives of strain vector with respect to nodal displacements [16]. The final form is

$$
\mathrm{B}=\left[\begin{array}{cccccc}
N_{1}^{\prime} \cos \omega & N_{1}^{\prime} \sin \omega & N_{1} \gamma & N_{2}^{\prime} \cos \omega & N_{2}^{\prime} \sin \omega & N_{2} \gamma \\
-N_{1}^{\prime} \sin \omega & N_{1}^{\prime} \cos \omega & -N_{1}(1+\epsilon) & -N_{2}^{\prime} \sin \omega & N_{2}^{\prime} \cos \omega & -(1+\epsilon) N_{2} \\
0 & 0 & N_{1}^{\prime} & 0 & 0 & N_{2}^{\prime}
\end{array}\right]
$$

where $\omega=\theta+\phi_{0}, \epsilon$ and $\gamma$ are evaluated by

$$
\begin{aligned}
& \epsilon=\frac{L \cos \psi \cos \theta+L \sin \psi \sin \theta}{L_{0}}-1 \\
& \gamma=\frac{L \sin \psi \cos \theta-L \cos \psi \sin \theta}{L_{0}}
\end{aligned}
$$

Here, $N_{1}^{\prime}=d N_{1} / d x$, and similarly for $N_{2}^{\prime}$. The above expressions for $\epsilon$ and $\gamma$ are the same as shown in Eq. 1, in which the strains were expressed in terms of displacement functions. Finally, the beam elemental potential energy based on the finite strain beam theory is

$$
U=\frac{1}{2} \int_{0}^{L_{0}}\left[E A(\bar{x}) \epsilon^{2}+G A(\bar{x}) \gamma^{2}+E I(\bar{x})\left(\frac{d \theta}{d \bar{x}}\right)^{2}\right] d \bar{x}
$$

where $G$ is the shear modulus. For isotropic materials, it can be expressed in terms of Young's modulus, $E$, and material constant Poisson ratio, $\nu$.

$$
G=\frac{E}{2(1+\nu)}
$$

Equation 11 can also be rewritten in a vector form.

$$
U=\frac{1}{2} \int_{0}^{L_{0}} P^{T} h d \bar{x}
$$

where the stress resultant vector $P$ is given by

$$
P=\left[\begin{array}{l}
E A(\bar{x}) \epsilon \\
G A(\bar{x}) \gamma \\
E I(\bar{x}) \theta^{\prime}
\end{array}\right]=D h
$$

and the material constant matrix, $D$, is defined as

$$
D=\left[\begin{array}{ccc}
E A(\bar{x}) & 0 & 0 \\
0 & G A(\bar{x}) & 0 \\
0 & 0 & E I(\bar{x})
\end{array}\right]
$$

The internal nodal force vector can be obtained by taking the first variation of the potential energy, Eq. 13, with respect to the nodal displacements.

$$
\delta U=\int_{0}^{L_{0}} P^{T} B d \bar{x} \delta q
$$

and the nodal internal force vector is

$$
f=\int_{0}^{L_{0}} B^{T} P^{T} d \bar{x}
$$

$f$ is a $6 \times 1$ vector. The tangent stiffness matrix can be defined by taking the first variation of internal force vector, as shown in Eq. 17.

$$
\delta f=\int_{0}^{L_{0}}\left(B^{T} \delta P^{T}+\delta B^{T} P^{T}\right) d \bar{x}=\left(k_{m}+k_{g}\right) \delta q=k \delta q
$$

The tangent stiffness matrix, $k$, is the sum of material stiffness, $k_{m}$, and geometric stiffness matrix, $k_{g}$. Recalled Eq. 14 and taking its variation with respect to nodal displacements, the material stiffness matrix is 


$$
k_{m}=\int_{0}^{L_{0}} B^{T} D B^{T} d \bar{x}
$$

In order to calculate the geometric stiffness matrix, $k_{g}$, the important step is to calculate the variation of the $B$ matrix with respect to nodal displacements. As shown in Eq. 9, the size of $B$ matrix is $3 \times 6$ and it is a function of $\epsilon, \gamma$, and $\theta$. Therefore, the $B$ matrix is a function of nodal displacement as well. The variation of $B$ with respect to the nodal displacements can be calculated as

$$
\delta B^{j}(k, i)=\frac{\partial B(k, i)}{\partial q_{j}} \delta q_{j}=R^{j}(k, i) \delta q_{j} \quad i, j=1,6 ; k=1,3
$$

where after mathematical manipulation, the geometric stiffness matrix, $k_{g}$, can be computed as

$$
k_{g}=\int_{0}^{L_{0}}\left(E A(\bar{x}) \epsilon B_{u}+G A(\bar{x}) \gamma B_{w}\right) d \bar{x}
$$

where $B_{u}$ and $B_{w}$ are $6 \times 6$ matrices, and they are assembled using Eq. 20, as below:

$$
\begin{aligned}
& B_{u}=\left[\begin{array}{llllll}
R^{1}(1,:)^{T} & R^{2}(1,:)^{T} & R^{3}(1,:)^{T} & R^{4}(1,:)^{T} & R^{5}(1,:)^{T} & R^{6}(1,:)^{T}
\end{array}\right] \\
& B_{w}=\left[\begin{array}{llllll}
R^{1}(2,:)^{T} & R^{2}(2,:)^{T} & R^{3}(2,:)^{T} & R^{4}(2,:)^{T} & R^{5}(2,:)^{T} & R^{6}(2,:)^{T}
\end{array}\right]
\end{aligned}
$$

We have obtained the elemental tangent stiffness, Eqs. 19 and 21, and nodal internal force vector, Eq. 17. In order to avoid shear locking [16,24,25], one Gaussian point was used to calculate the elemental tangent stiffness matrices and equivalent internal force vector. The Newton-Raphson method was used to solve for the static nonlinear responses [21].

In order to account for the dynamics of a fly rod during a cast, we included appropriate inertia terms in our nonlinear finite element model. Since the above nonlinear finite element model was developed with respect to the fixed frame, we simply include inertia terms without the linearizations and simplification as used by Gatti [6]. The kinetic energy of the fly rod is

$$
\mathrm{T}=\frac{1}{2} \int_{0}^{\mathrm{L}_{0}}\left[\rho \mathrm{A}(\overline{\mathrm{x}})\left(\frac{\partial \mathrm{u}}{\partial \mathrm{t}}\right)^{2}+\rho \mathrm{A}(\overline{\mathrm{x}})\left(\frac{\partial \mathrm{w}}{\partial \mathrm{t}}\right)^{2}+\rho \mathrm{I}(\overline{\mathrm{x}})\left(\frac{\partial \theta}{\partial \mathrm{t}}\right)^{2}\right] \mathrm{d} \overline{\mathrm{x}}
$$

where the $\rho$ is the density of fly rod material. Using the same interpolation functions for axial displacement, $u(\bar{x})$, transverse displacement, $w(\bar{x})$, and rotation angular displacement, $\theta(\bar{x})$, as shown in Eq. 2, we obtain the elemental mass matrix, which is a $6 \times 6$ matrix.

$$
\mathrm{m}=\int_{0}^{\mathrm{L}_{0}} \rho\left[\begin{array}{cccccc}
A(\bar{x}) N_{1}^{2} & 0 & 0 & A(\bar{x}) N_{1} N_{2} & 0 & 0 \\
0 & A(\bar{x}) N_{1}^{2} & 0 & 0 & A(\bar{x}) N_{1} N_{2} & 0 \\
0 & 0 & I(\bar{x}) N_{1}^{2} & 0 & 0 & I(\bar{x}) N_{1} N_{2} \\
A(\bar{x}) N_{1} N_{2} & 0 & 0 & A(\bar{x}) N_{2}^{2} & 0 & 0 \\
0 & A(\bar{x}) N_{1} N_{2} & 0 & 0 & A(\bar{x}) N_{2}^{2} & 0 \\
0 & 0 & I(\bar{x}) N_{1} N_{1} & 0 & 0 & I(\bar{x}) N_{2}^{2}
\end{array}\right] \mathrm{d} \overline{\mathrm{x}}
$$

After assembly, the total mass matrix, $M$, is obtained. The next question is how to solve for the nonlinear dynamic response. As discussed by Simo and Vu-Quoc [16], the Newmark algorithm [26], which is a well-known predictorcorrector form, including the Newton-Raphson equilibrium iteration loop, can be used in the dynamic nonlinear finite element analysis. The convergence of this approach for nonlinear dynamic simulations has been well discussed by Belytschko and Hughes [27] and Argyris and Mlejnek [28]. The algorithm used in here is shown as follows:

Step 1. Initialize $i, i=0$

Step 2. Predictor

$$
\begin{aligned}
& U_{t+\Delta t}^{i}=U_{t} \\
& \ddot{U}_{t+\Delta t}^{i}=-\frac{1}{\beta \Delta t} \dot{U}_{t}+\left(1-\frac{1}{2 \beta}\right) \ddot{U}_{t} \\
& \dot{U}_{t+\Delta t}^{i}=\dot{U}_{t}+\Delta t\left[(1-\gamma) \ddot{U}_{t}+\gamma \ddot{U}_{t+\Delta t}^{i}\right]
\end{aligned}
$$


Step 3. Increment $i, i=i+1$

Step 4. Calculate effective stiffness, $K_{e f f}^{i}$, and residual force vector, $Y^{i}$

$$
\begin{aligned}
K_{e f f}^{i} & =\frac{1}{\beta \Delta t^{2}} M^{i-1}+\frac{\gamma}{\beta \Delta t} D^{i-1}+K_{m}^{i-1}+K_{g}^{i-1} \\
Y^{i} & =R_{e x t}^{i}-M^{i-1} \ddot{U}_{t+\Delta t}^{i-1}-D^{i-1} \dot{U}_{t+\Delta t}^{i-1}-F_{t+\Delta t}^{i-1}
\end{aligned}
$$

Step 5. Solve for displacement increment, $\Delta U^{i}=\left(K_{e f f}^{i}\right)^{-1} Y^{i}$

Step 6. Corrector

$$
\begin{aligned}
& U_{t+\Delta t}^{i}=U_{t+\Delta t}^{i-1}+\Delta U^{i} \\
& \dot{U}_{t+\Delta t}^{i}=\dot{U}_{t+\Delta t}^{i-1}+\frac{\gamma}{\beta \Delta t} \Delta U^{i} \\
& \ddot{U}_{t+\Delta t}^{i}=\ddot{U}_{t+\Delta t}^{i-1}+\frac{1}{\beta \Delta t^{2}} \Delta U^{i}
\end{aligned}
$$

Step 7. If $\left\|Y^{i}\right\|>1.0 \times 10^{-5}$, repeat iteration, go to step 4, else $t=t+\Delta t$ and go to Step 1 .

The parameter values of $\beta=0.25$, and $\gamma=0.5$ were used in our calculation, and the $D$ matrix the is damping matrix for the fly rod system. We assume that $D$ is the Rayleigh damping matrix and it is expressed by

$$
D=\eta M
$$

Where $\eta$ is a constant. In order to obtain the convergent and accurate solution, the time step size should be as small, say $\Delta t \leqslant 1.0 \times 10^{-3}$.

\section{Fly rod casting dynamics}

As discussed by Gatti [6], the coupled and uncoupled fly rod/line system produced similar results. Fly rod tip path becomes very important because it serves as the boundary condition that dictates fly line motions during a cast. We have developed a nonlinear finite element model to account for the transient response of a fly rod during a cast. The next step is to develop a fly line model.

\subsection{Fly line model}

Figure 2 showed a schematic representation of fly rod and line motion during an overhead cast [5]. The sequence runs from (a) to (f). First, the angler raises the rod to lift the line and to throw it behind. The line will load the rod until it is finally parallel to the ground, as shown in Fig. 2 (d). The next stage is forward casting. The angler applies a torque to rotate the rod and the line moves forward lead by the fly rod tip. The angler abruptly stops the rod. The line attached to the tip will stop as well. In this case, a loop will be formed, having three distinct segments in the line. These are the upper traveling line, the front loop, and the lower stationary line. Spolek [3] used the work and energy balance method to calculate fly velocity at end of the line by specifying the line shapes for different segments. The traveling and stationary line segments were modeled as straight and parallel to the horizontal direction, and the front loop had a semi-circular shape. However, we want to know more details of line motion during a cast, especially presentation of the fly onto the water. Robson [5] presented a lumped parameter model to capture the fly line motion and validated his results using casting data. We also adopted the lumped parameter approach in this study. Gatti [6, 7] also applied the continuum cable model for fly line analysis. In all of these analyses [3,5-7] including our own, the casting simulation starts at stage (d) in Fig. 2, in which the line is parallel to the ground and with zero velocity for both fly rod and line. The fly line motions were confined to a vertical plane only.

Figure 3 shows the fly line lumped parameter model. The fly line was represented by a series of rigid cylinders, where adjacent elements were connected by a massless hinge. Gravity and air resistance forces were applied to these 


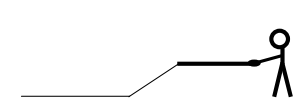

(a)

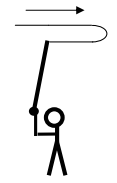

(c)

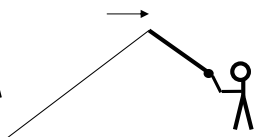

(b)

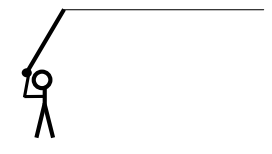

(d)

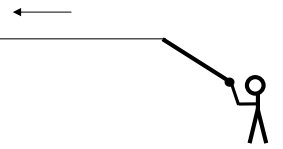

(f)

Fig. 2. A typical forward casting sequence.

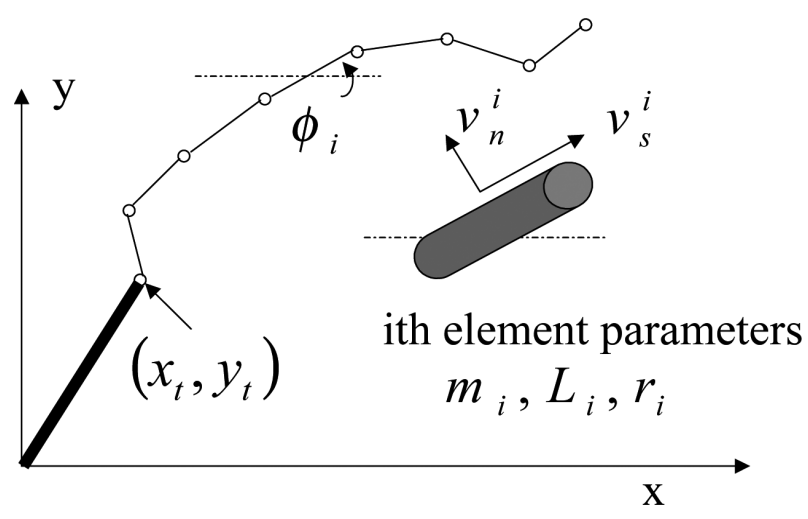

Fig. 3. A lumped parameter fly line model.

line filaments during a cast. For each line element, there is only one degree of freedom: the rotational angle with respect to the ground [5]. We modified Robson's model slightly, by using the rotational angle measured at the center of each line element instead of the rotational angle at the leading tip of the cylinder,. For the $i t h$ filament of the line system, as shown in Fig. 3, the position is

$$
\begin{aligned}
& x_{i}=x_{t}+\sum_{j=1}^{i} L_{i}\left(1-\frac{1}{2} \delta_{i j}\right) \cos \phi_{i} \\
& y_{i}=y_{t}+\sum_{j=1}^{i} L_{i}\left(1-\frac{1}{2} \delta_{i j}\right) \sin \phi_{i}
\end{aligned}
$$

where $\delta_{i j}$ is the Kronecker delta function. $x_{t}$ and $y_{t}$ are fly rod tip position in the $x$ and $y$ directions, as shown in Fig. 3, $L_{i}$ is the $i t h$ line element, $\phi_{i}$ is the $i t h$ element rotation angle measured with respect to horizontal direction. Velocity and acceleration of each fly line element can be calculated by differentiating position with respect to time.

The kinetic energy of the fly line system is:

$$
T=\frac{1}{2} \sum_{i=1}^{n} m_{i}\left({\dot{x_{i}}}^{2}+{\dot{y_{i}}}^{2}\right)
$$


and its potential energy is

$$
V=\sum_{i=1}^{n} m_{i} g y_{i}
$$

Here, $n$ is the total number of line elements, $m_{i}$ is the mass of each element, and $g$ is gravitational acceleration. We utilized Rayleigh's dissipation function [29], $\Psi$, to include the effects of air resistance on fly line elements,

$$
\Psi=\frac{1}{3} \sum_{i=1}^{n}\left(C_{D} \rho_{a} r_{i} L_{i}\left(v_{n}^{i}\right)^{3}+C_{s} \rho_{a} \pi r_{i} L_{i}\left(v_{s}^{i}\right)^{3}\right)
$$

where $\rho_{a}$ is the air density, $r_{i}$ is the radius of $i$ th line element, $v_{D}^{i}$ and $v_{s}^{i}$ are the velocity components along normal and tangent direction of $i t h$ line element, respectively, as shown in Fig. 3. They can be expressed by $\dot{x}_{i}, \dot{y}_{i}$, and $\phi_{i}$.

$$
\begin{gathered}
v_{D}^{i}=-\dot{x}_{i} \sin \phi_{i}+\dot{y}_{i} \cos \phi_{i} \\
v_{S}^{i}=\dot{x}_{i} \cos \phi_{i}+\dot{y}_{i} \sin \phi_{i}
\end{gathered}
$$

and $C_{D}$ and $C_{S}$ are drag coefficients, which were discussed by Robson [5]. $C_{S}$ was assumed to be constant: $C_{S}=$ 0.005. Additional details of $C_{S}$ can be found in [3]. The pressure drag coefficient, $C_{D}$, depends on line velocity and is a function of Reynolds number, Re, [5], as below:

$$
\begin{aligned}
& C_{D}=7.16 \quad R e<1 \\
& C_{D}=7.16 R^{-0.42 \quad} \quad 1<R e<34 \\
& C_{D}=3.02 R^{-0.165 \quad} \quad 34<R e<1580 \\
& C_{D}=0.9 \quad R e>1580
\end{aligned}
$$

where for each line element, $R e$ is determined by

$$
R e=1.364 \times 10^{5} r_{i} \sqrt{{\dot{x_{i}}}^{2}+{\dot{y_{i}}}^{2}}
$$

Lagrange's Equations in this case become [29],

$$
\frac{d}{d t}\left(\frac{\partial(T-V)}{\partial \dot{\phi}_{k}}\right)-\frac{\partial(T-V)}{\partial \phi_{k}}+\frac{\partial \Psi}{\partial \dot{\phi}_{k}}=0 \quad k=1,2, \ldots, n
$$

resulting in $n$ differential equations in terms of the $n$ unknown rotation angles,

$$
\begin{array}{rcc}
a_{11} \ddot{\phi}_{1}+a_{12} \ddot{\phi}_{2}+\cdots+a_{1 n} \ddot{\phi_{n}} & =Q_{1} \\
\vdots & \vdots & \vdots \\
a_{i 1} \ddot{\phi}_{1}+a_{i 2} \ddot{\phi}_{2}+\cdots+a_{i n} \ddot{\phi_{n}} & = & Q_{i} \\
\vdots & \vdots & \vdots \\
a_{n 1} \ddot{\phi_{1}}+a_{n 2} \ddot{\phi_{2}}+\cdots+a_{n n} \ddot{\phi_{n}} & = & Q_{n}
\end{array}
$$

The coefficient, $a_{i j}$, is

$$
a_{i j}=\left\{\begin{array}{cc}
\sum_{k=i}^{n} m_{k} l_{k}^{2}\left(1-\frac{3}{4}\right) \delta_{i k} & i=j \\
A_{i j} L_{i} L_{j} \cos \left(\phi_{i}-\phi_{j}\right) & i \neq j
\end{array}\right.
$$

and the coefficient $A_{i j}$ is defined as

$$
A_{i j}= \begin{cases}\sum_{k=j}^{n} m_{k}-\frac{1}{2} m_{j} & j>i \\ \sum_{k=i}^{n} m_{k}-\frac{1}{2} m_{i} \quad j \leqslant i\end{cases}
$$

The right hand term, $Q_{i}$, is 


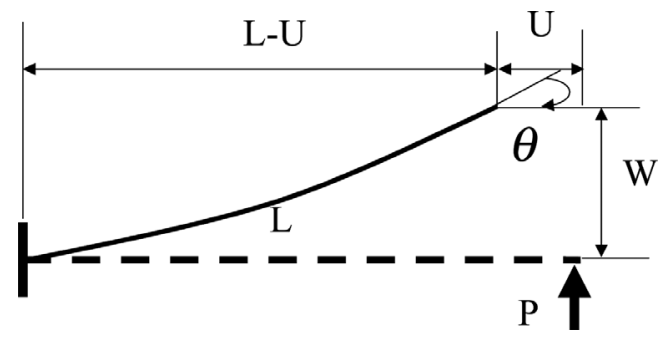

Fig. 4. A cantilever beam under tip point force.

$$
\begin{aligned}
Q_{i}= & -\sum_{k=i}^{n} m_{k}\left(1-\frac{1}{2} \delta_{i k}\right) L_{i}\left(g \cos \phi_{i}-\sin \phi_{i} \ddot{x}_{t}+\cos \phi_{i} \ddot{y}_{t}\right) \\
& +\sum_{k=1 \neq i}^{n} A_{i k} L_{i} L_{k}\left(\dot{\phi_{k}}\right)^{2} \sin \left(\phi_{k}-\phi_{i}\right) \\
& -\sum_{k=i}^{n} C_{D}^{k} \rho_{a} r_{k} L_{k} v_{n}^{k}\left\|v_{n}^{k}\right\|\left(1-\frac{1}{2} \delta_{i k}\right) L_{i} \cos \left(\phi_{k}-\phi_{i}\right) \\
& -\sum_{k=i}^{n} C_{S} \rho_{a} \pi r_{k} L_{k} v_{s}^{k}\left\|v_{s}^{k}\right\|\left(1-\frac{1}{2} \delta_{i k}\right) L_{i} \sin \left(\phi_{i}-\phi_{k}\right)
\end{aligned}
$$

The governing equations of the fly line model are second order differential equations, as shown below in a vector form

$$
[a] \ddot{\phi}=[Q]
$$

Rewriting as a first order differential form:

$$
\{\dot{Y}\}=\left[\begin{array}{cc}
I_{n \times n} & 0_{n \times n} \\
0_{n \times n} & {[a]^{-1}}
\end{array}\right]\left\{\begin{array}{c}
\dot{\phi} \\
Q
\end{array}\right\}
$$

The above equation is a state space model, and $\mathbf{Y}$ is a state vector containing the rotational displacements and velocity. It is

$$
\{Y\}=\left\{\begin{array}{c}
\phi \\
\dot{\phi}
\end{array}\right\}
$$

For a giving time step $\Delta t$ and fly rod tip information, Eq. 32 can be easily solved by using a numerical integration scheme, such as a Runge-Kutta 4th order method [30].

\section{Results}

\subsection{Static nonlinear deflection}

In order to validate our nonlinear finite element model, we considered a tapered cantilevered beam, as shown in Fig. 4. Fertis [31] presented the exact solutions for tapered beam under tip point force by integrating the beam nonlinear differential equations. The beam was 25.4 meters (1000 in.) long, and bending stiffness, $E I$, was assumed to be $E I=516.21 N-m^{2}\left(180 \times 10^{3} k i p-i n^{2}\right)$. The variations of beam moment of inertia and cross section areas were defined as

$$
\begin{gathered}
E I(x)=E I_{0}\left(r+\frac{1-r}{L} x\right)^{3} \\
A(x)=A_{0}\left(r+\frac{1-r}{L} x\right)
\end{gathered}
$$


Table 1

Tip vertical displacement results for a tapered cantilevered beam under tip point force

\begin{tabular}{cccc}
\hline $\begin{array}{c}\text { Taper Parameter } \\
r\end{array}$ & $\begin{array}{c}\text { Exact } \\
W[\mathrm{~m}]\end{array}$ & $\begin{array}{c}\text { NFEM } \\
W[\mathrm{~m}]\end{array}$ & $\begin{array}{c}\text { Error } \\
{[\%]}\end{array}$ \\
\hline 1.0 & 18.594 & 18.586 & -0.04 \\
1.2 & 16.302 & 16.303 & 0.00 \\
1.4 & 13.990 & 13.995 & 0.03 \\
1.5 & 12.881 & 12.885 & 0.04 \\
1.6 & 11.822 & 11.827 & 0.05 \\
1.8 & 9.900 & 9.905 & 0.05 \\
2.0 & 8.264 & 8.270 & 0.08 \\
2.2 & 6.910 & 6.916 & 0.09 \\
2.5 & 5.331 & 5.337 & 0.10 \\
3.0 & 3.580 & 3.585 & 0.13 \\
\hline
\end{tabular}

Table 2

Tip horizontal displacement results for a tapered cantilevered beam under tip point force

\begin{tabular}{cccc}
\hline $\begin{array}{c}\text { Taper Parameter } \\
r\end{array}$ & $\begin{array}{c}\text { Exact } \\
U[\mathrm{~m}]\end{array}$ & $\begin{array}{c}\text { NFEM } \\
{[\mathrm{m}]}\end{array}$ & $\begin{array}{c}\text { Error } \\
{[\%]}\end{array}$ \\
\hline 1.0 & 10.540 & 10.518 & -0.22 \\
1.2 & 7.728 & 7.714 & -0.18 \\
1.4 & 5.510 & 5.500 & -0.17 \\
1.5 & 4.618 & 4.610 & -0.18 \\
1.6 & 3.859 & 3.852 & -0.18 \\
1.8 & 2.684 & 2.678 & -0.22 \\
2.0 & 1.869 & 1.864 & -0.24 \\
2.2 & 1.312 & 1.309 & -0.30 \\
2.5 & 0.791 & 0.788 & -0.41 \\
3.0 & 0.368 & 0.365 & -0.71 \\
\hline
\end{tabular}

Table 3

Tip angular displacement results for a tapered cantilevered beam under tip point force

\begin{tabular}{cccc}
\hline $\begin{array}{c}\text { Taper Parameter } \\
r\end{array}$ & $\begin{array}{c}\text { Exact } \\
\theta[\mathrm{deg} .]\end{array}$ & $\begin{array}{c}\text { NFEM } \\
\theta[\mathrm{deg} .]\end{array}$ & $\begin{array}{c}\text { Error } \\
{[\%]}\end{array}$ \\
\hline 1.0 & 71.97 & 72.11 & 0.20 \\
1.2 & 63.67 & 63.82 & 0.24 \\
1.4 & 55.37 & 55.54 & 0.31 \\
1.5 & 51.42 & 51.59 & 0.32 \\
1.6 & 47.65 & 47.82 & 0.35 \\
1.8 & 40.65 & 40.95 & 0.73 \\
2.0 & 34.89 & 35.05 & 0.44 \\
2.2 & 29.93 & 30.08 & 0.50 \\
2.5 & 24.01 & 24.15 & 0.60 \\
3.0 & 17.17 & 17.31 & 0.83 \\
\hline
\end{tabular}

where $r$ is taper parameter, and cross section areas is assumed as $A_{0}=32.258 \mathrm{~cm}^{2}\left(5 \mathrm{in}^{2}\right)$, where Poisson's ratio is $\nu=0$. For a fixed tip loading, $P=4448.22 \mathrm{~N}(1 \mathrm{kip})$, we calculated tip deflection under different taper rates, or a taper parameter $r$ varying from 1.0 to 3.0. Our predictions of tip displacements were compared to those obtained by Fertis [31], as shown in Table 4.1 to 3. These results are transverse, horizontally, and rotational displacements at tip. The maximum error of our tip deflection predictions is less than $1 \%$. Therefore, the nonlinear finite element model was validated analytically and the results compared well with the exact solutions by Fertis [31]. Further comparison was conducted to compare the deformed configuration of a uniform cantilever beam subjected to a transverse end load as studied by Pai [32] (example 5.3 on page 349), where a nonlinear beam theory was used to obtain the solution. Good correlation was achieved as shown in Fig. 5. An experimental fly rod was made as shown in Fig. 6 in order to validate our nonlinear finite element experimentally. It was a three-piece fly rod. Table 4 shows the dimensions of each piece, along with its respective Young's modulus $E_{b}, E_{m}$ and $E_{t}$. The Young's modulus were determined experimentally via a bending strain test. The Poisson's ratio was assumed to be $\nu=0$ and density was $\rho=1600 \mathrm{~kg} / \mathrm{m}^{3}$. A tip load was applied to our experimental fly rod and tip deflection was measured. Figure 7 shows the tip load-deflection curve. Both linear and nonlinear finite element analyses were compared to experimental data. Because a fly rod is very slender, long, and flexible beam, geometric nonlinear effects exist. Linear analysis diverges from experimental data when applied tip mass exceed 50 gram. Our nonlinear finite element results matched well with the experimental data for the entire range of applied tip mass tested up to $150 \mathrm{~g}$.

\subsection{Fly rod casting simulations}

As discussed by Robson [5], a reasonable approximation to a normal forward cast appears to be a linearly increasing acceleration followed by a rapid exponential decay of the angular velocity after the attempted stop. Thus, a cast can be described using following parameters: initial butt angle, $\theta_{1}$, corresponding to $t=0$, attempted stop angle, $\theta_{2}$, 


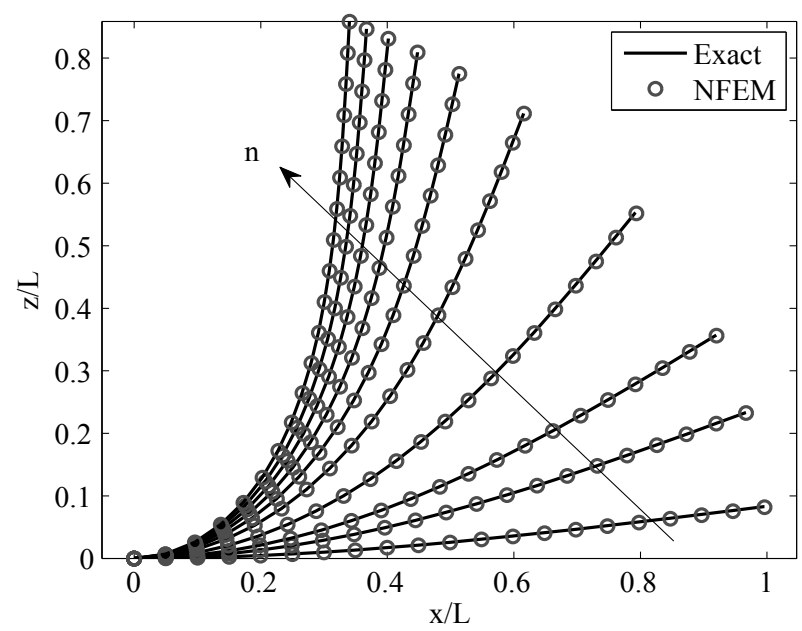

Fig. 5. Deformed configuration of a cantilever beam subjected to a transverse end load [32], where $F=n \frac{\pi^{2} E I}{4 L^{2}}$ and $n=[0.1,0.3,0.5,1,2,3$, $4,5,6,7]$.

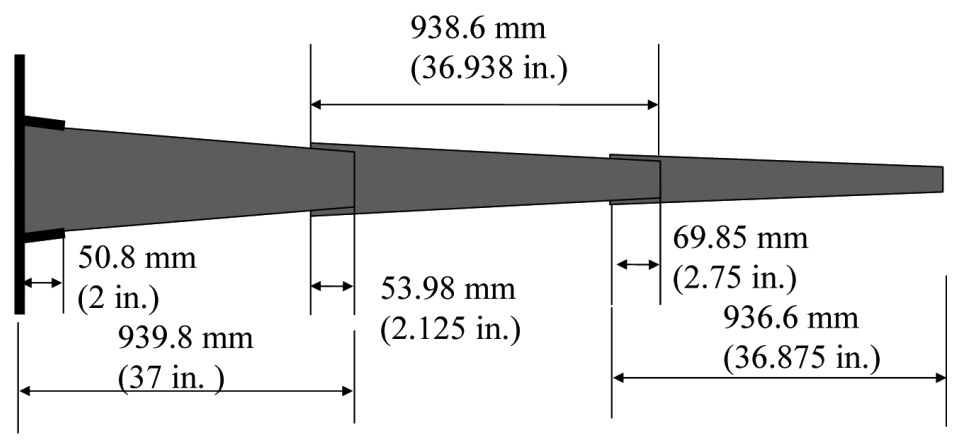

Fig. 6. Experimental 3 piece assembled fly rod.

corresponding to $t=t_{2}$, rate of increase of angular acceleration, $\alpha$, between these two angles, and stopping time constant, $T_{w}$. He presented the empirical formulations to describe the butt end motion of a fly rod and the results were validated using a film record of some actual casts. We adopted his approach in our casting simulations. The expressions used for butt end angle, $\theta$, during a cast were

$$
\theta=\left\{\begin{array}{cc}
\theta_{1}+\frac{\alpha t^{3}}{6} & t \leqslant t_{2} \\
\theta_{2}+\frac{\alpha t_{2}^{2}}{2} T_{w}\left(1-e^{\frac{\left(t_{2}-t\right)}{T_{w}}}\right) & t>t_{2}
\end{array}\right.
$$

Then the angular velocity and acceleration can be obtained

$$
\begin{aligned}
& \dot{\theta}=\left\{\begin{array}{cc}
\frac{\alpha t^{2}}{2} & t \leqslant t_{2} \\
\frac{\alpha t_{2}^{2}}{2} e^{\frac{\left(t_{2}-t\right)}{T_{w}}} & t>t_{2}
\end{array}\right. \\
& \ddot{\theta}=\left\{\begin{array}{cc}
\alpha t & t \leqslant t_{2} \\
-T_{w} \frac{\alpha t_{2}^{2}}{2} e^{\frac{\left(t_{2}-t\right)}{T_{w}}} & t>t_{2}
\end{array}\right.
\end{aligned}
$$

where we assumed that

$$
t_{2}=\left[\frac{6\left(\theta_{2}-\theta_{1}\right)}{\alpha}\right]^{\frac{1}{3}}
$$


Table 4

Fly rod dimensions and material properties(O., outer, I., inner; D., diameter)

\begin{tabular}{|c|c|c|c|c|c|c|c|}
\hline \multirow[t]{2}{*}{ Section } & \multirow{2}{*}{$\begin{array}{l}\text { Length } \\
{[\mathrm{mm}]}\end{array}$} & \multicolumn{2}{|c|}{ Bottom } & \multicolumn{2}{|c|}{ Top } & \multirow{2}{*}{$\begin{array}{c}\text { Ferrule } \\
\text { OD } \\
{[\mathrm{mm}]}\end{array}$} & \multirow{2}{*}{$\begin{array}{c}\text { Young's Modulus } \\
\text { [Gpa }]\end{array}$} \\
\hline & & $\begin{array}{c}\text { ID } \\
{[\mathrm{mm}]}\end{array}$ & $\begin{array}{c}\mathrm{OD} \\
{[\mathrm{mm}]}\end{array}$ & $\begin{array}{c}\text { ID } \\
{[\mathrm{mm}]}\end{array}$ & $\begin{array}{c}\mathrm{OD} \\
{[\mathrm{mm}]}\end{array}$ & & \\
\hline Butt & 939.80 & 10.97 & 9.14 & 7.29 & 5.49 & - & 97.6 \\
\hline Middle & 938.23 & 9.20 & 7.72 & 4.25 & 3.01 & 8.28 & 112.4 \\
\hline Tip & 936.63 & 61.0 & 4.72 & 1.68 & 0.76 & 5.13 & 147 \\
\hline
\end{tabular}

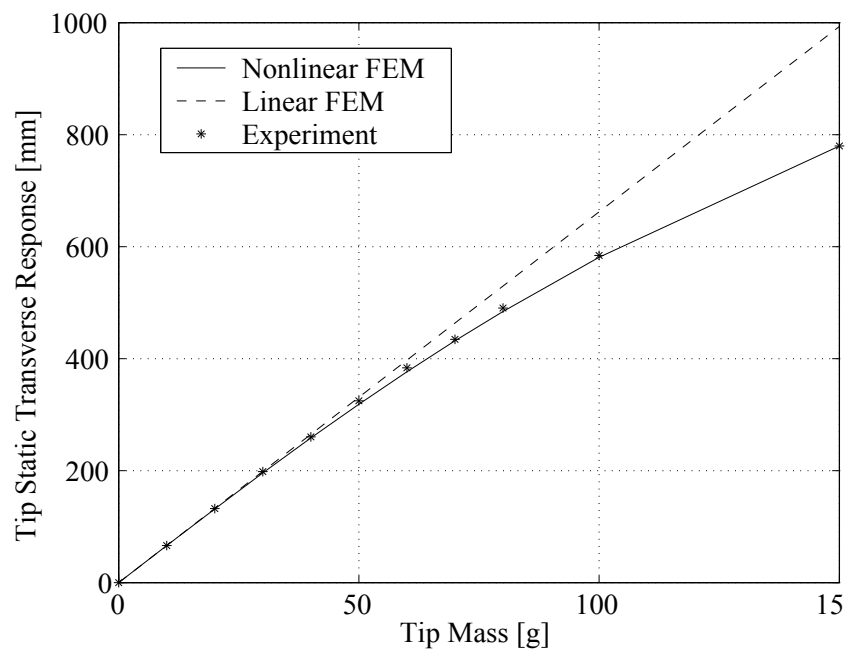

Fig. 7. Fly rod tip load and vertical deflection curve.

The input parameters of the rod butt motion during a cast are $\theta_{1}=75^{\circ}, \theta_{2}=130^{\circ}, T_{w}=0.03 \mathrm{~s}$, and $\alpha=100,000^{\circ} / s^{3}$.

Figure 8 plotted the time history of the butt end during a cast. For a rigid fly rod, the fly rod tip position, velocity, and acceleration, at any time $t$ can be calculated using above equations. Then, fly line motions can be specified at any time $t$ using Eq. 31. For a flexible fly rod, we need to determine the fly rod profile during a cast. In this case, the transient response of a fly rod can be calculated based on the specified butt end motions. When the fly rod butt end reaches the stopping angle, $\theta_{2}$, we introduce the critical damping to fly rod system in order to simulate the braking mechanism of hand motion by angler, otherwise the fly rod will undergo finite rotation in our numerical simulations. Our goal is to compare to the case of rigid rod. In a real casting case, the fly rod will undergo small oscillation due to the under-damped characteristics of fly rod. Figure 9 shows the deformed shapes of our experimental fly rod, where the time step was set to be $0.0001 \mathrm{~s}$. Two casting simulations are discussed. One is for a rigid rod case and the second is for a flexible rod case. Each uses the same butt end motions and the rigid rod has same length as the flexible rod which is $2.69 \mathrm{~m}$. The fly line was an end tapered line with circular cross sectional area. The density of the line was $\rho_{l}=1158 \mathrm{~kg} / \mathrm{m}^{3}$. It had a constant radius for a length of 8 meter, and 2 meter tapered line. Total 30 line elements were used and average radius was accounted for tapered line section. The time step was set to be $0.001 \mathrm{~s}$ in the fly line simulations. The geometric details of the fly line are shown in Fig. 10.

Figures 11 and 12 show the results of a fly casting simulation with a rigid rod. As shown in Fig. 11, the simulation generated a loop as shown in [5]. The velocity time history of the first and last fly line elements are plotted in Fig. 12. The first line element reached its maximum velocity right after the rod attempted to stop at $\theta_{2}$, while the velocity of the last line element kept increasing and reached its maximum around $t=0.40 \mathrm{~s}$.

Figures 13 and 14 show the results of fly casting simulated using a flexible rod. The fly rod tip path, velocity, and acceleration information were used as the boundary conditions in order to simulate the fly line motions. As shown in Fig. 13, similar to the rigid rod case, a loop was generated and then propagated. However, the loop height was smaller than that for the rigid rod case, which suggest that less energy was dissipated during casting, because the drag loss results mostly from the loop [3]. In the flexible rod case, the first line element velocity profiles were similar to 


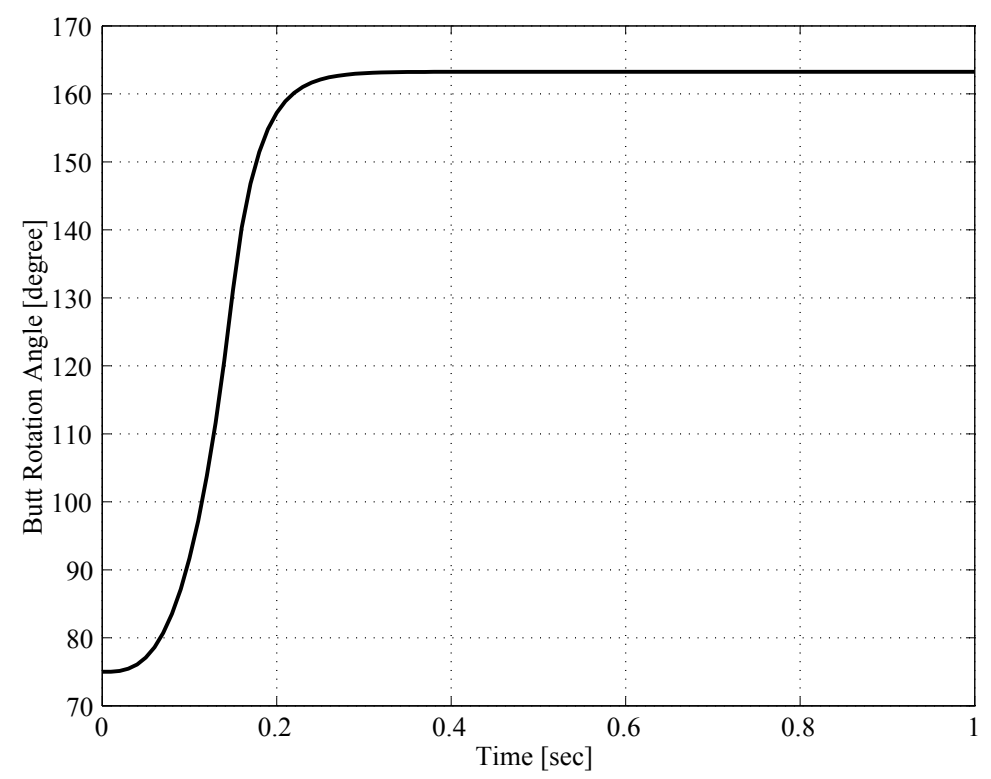

Fig. 8. Fly rod butt end motions in a cast.

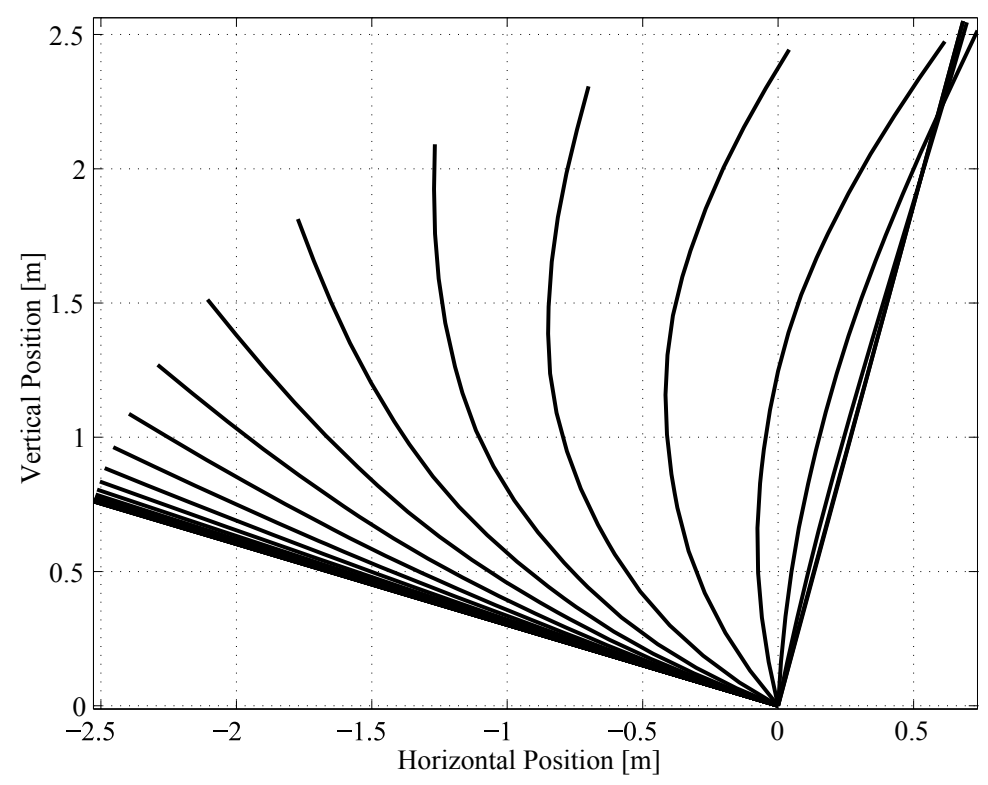

Fig. 9. Deformed shapes of a fly rod under butt end rotation.

those of the rigid rod case. However, the last line element velocity continued to increase until it reached its maximum around $t=0.7 \mathrm{~s}$, as shown in Fig. 14, which implied that this element undergoes acceleration throughout the cast and a much longer time interval than for the rigid rod case $(t=0.4 \mathrm{~s})$. The fly line last element velocity distribution in horizontal and vertical direction were different in the rigid case. The vertical velocity component, $V_{y}$, was almost zero before it reached the maximum speed, which is ideal case for a successful cast. A successful cast maintains the rod tip path in a straight line [2], so that the horizontal velocity dominates over vertical velocity component the line is propelled forward and straight. Based on our simulation results, we must include the flexibility of the fly rod in order to obtain the most accurate results, because the rod tip motion plays a significant role in determining the fly line motion. 


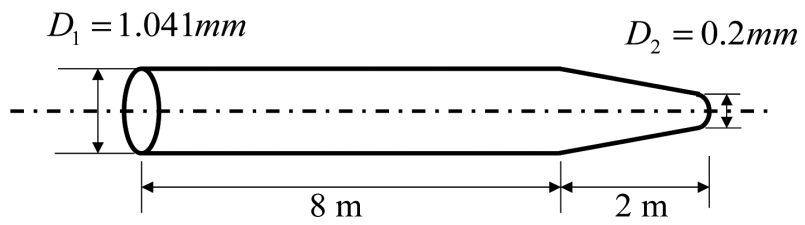

Fig. 10. Geometries of fly line.

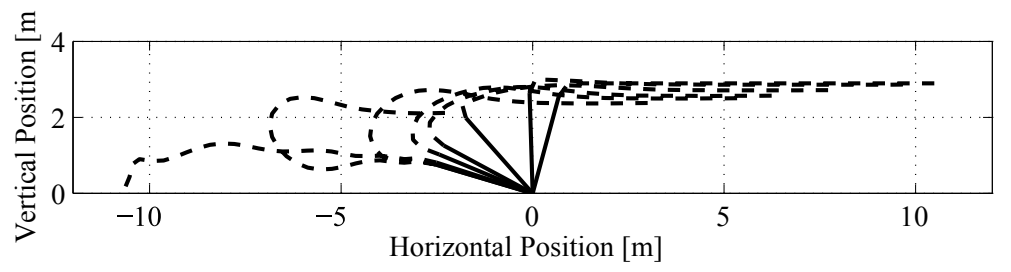

Fig. 11. Fly rod/line time history during a cast, where $t=[0,0.1,0.15,0.18,0.2,0.25,0.35,0.5] \mathrm{s}$, and rigid rod butt at origin, [Rigid Rod Case].
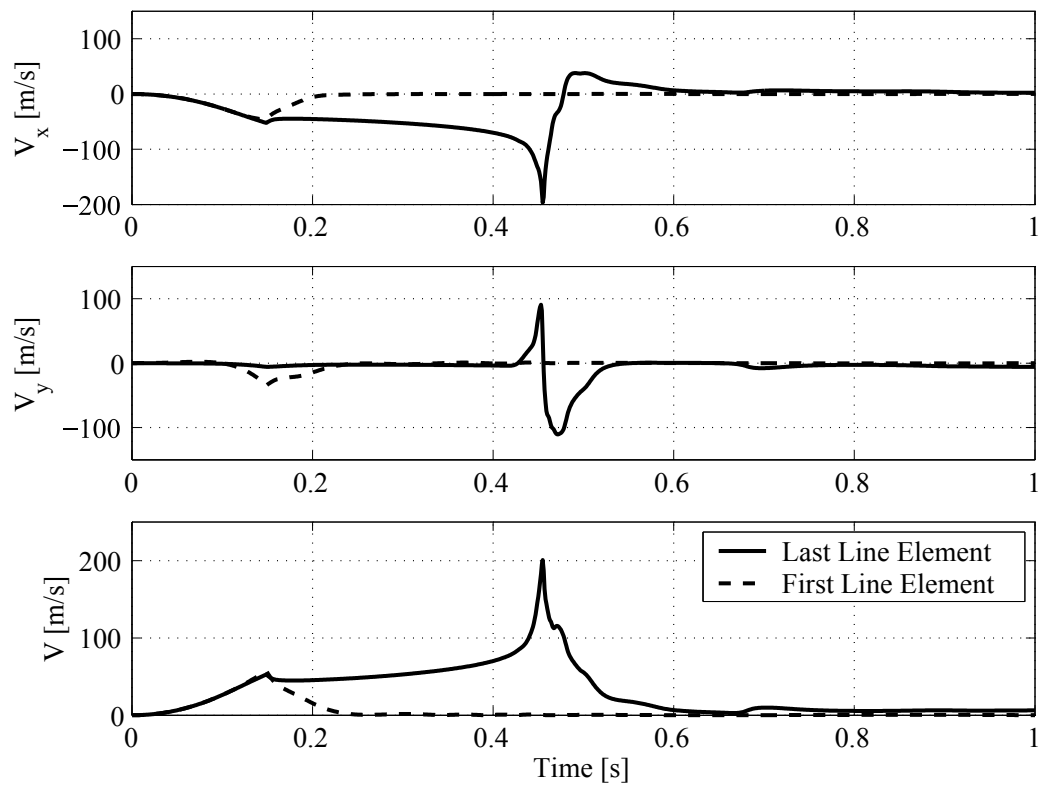

Fig. 12. Fly line first/last element velocity time history during a cast [Rigid Rod Case].

\section{Conclusions}

In this study, we developed an analysis of fly fishing rod casting dynamics. The nonlinear finite element model was used to analyze fly rod. The fly casting simulations were also investigated using Robson's fly line model, in which both rigid and flexible rods were considered. Thus, we have developed an engineering analysis to evaluate fly rod mechanics and fly rod/line casting dynamics.

Our predictions of fly rod static load-deflection curves were validated using both analytical results in literature and experiment data. In Tables 4.1 through 3, we compared our nonlinear finite element predictions to analytical results [31], where three tip vertical, horizontal and rotational displacements were calculated due to a tip force loading and different taper rates were assumed. Our maximum prediction error among these three displacements is less than $1 \%$ compared to analytical results for all cases of different tapered beam configurations. Also our predictions of the tip load-deflection curves for a graphite-epoxy composite fly fishing rod agreed well with experimental data, as shown in Fig. 7. We applied our nonlinear finite element model to transient response analysis of our experimental fly 


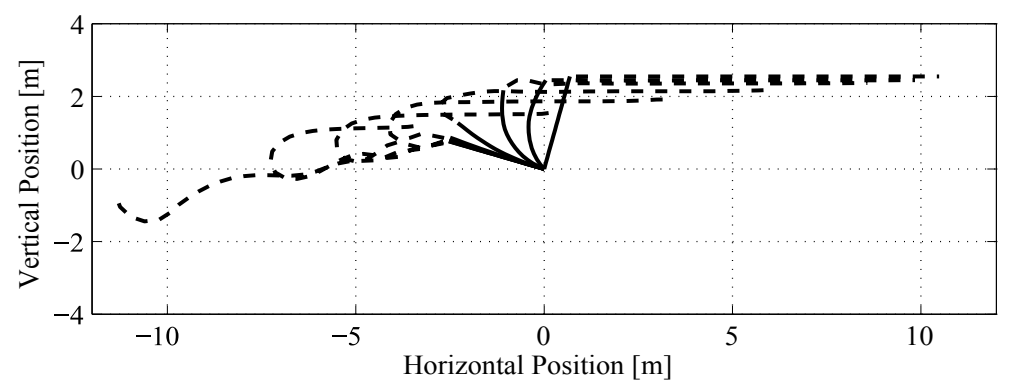

Fig. 13. Fly rod/line time history during a cast, where $t=[0,0.15,0.2,0.3,0.4,0.5,0.6,0.75] \mathrm{s}$, and fly rod butt at origin [Flexible Rod Case].
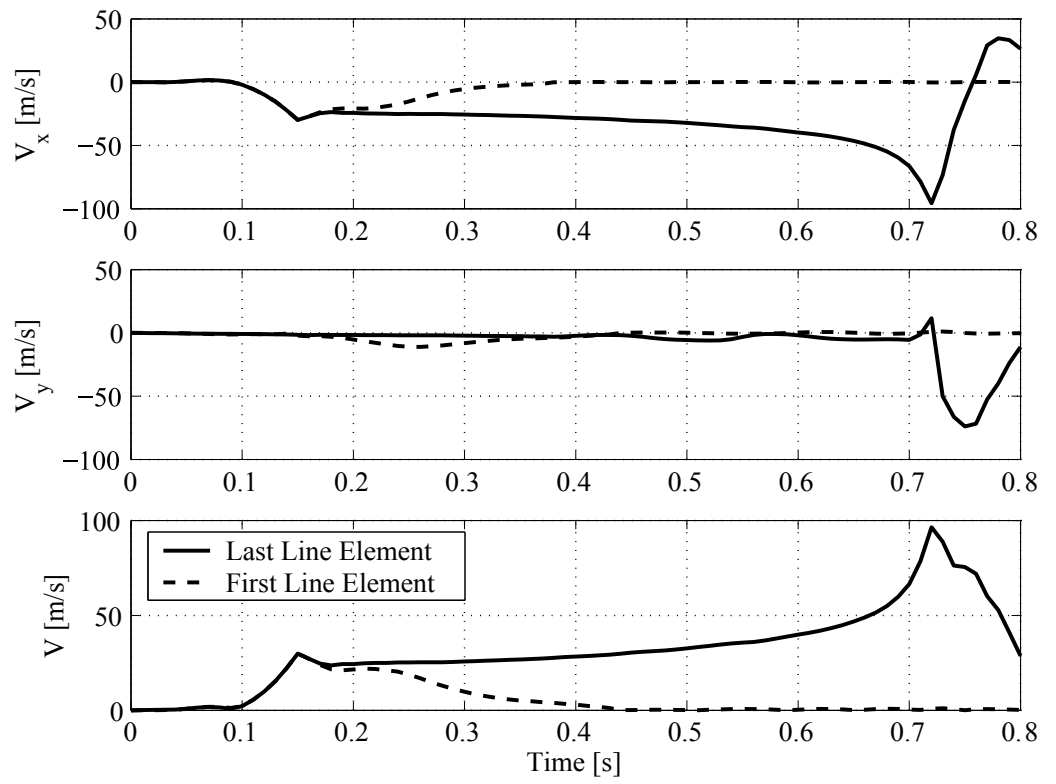

Fig. 14. Fly line first/last element velocity time history during a cast [Flexible Rod Case].

fishing rod under a forward casting stroke. The deformed shape of the fly rod was plotted in Fig. 9 during the cast. Our fly casting simulations were studied for both rigid and flexible fly rods. Introduction of rod flexibility greatly modified fly line behavior, so flexibility must be modeled in simulation of casting dynamics. Also, the flexible fly rod results resembled actual casting, which implies that fly rod flexibility properties must be included in order to simulate the actual casting scenario.

\section{Acknowledgement}

This work was supported by a Maryland Industrial Partnerships (MIPS) grant from the Maryland Technology Enterprise Institute (MTECH), and Beaverkill Rod Co. The author thanks Steve Forrer, President, Beaverkill Rod Co., for valuable discussions on fly rod and casting dynamics.

\section{References}

[1] Ernest G. Schwiebert, Trout, Dutton Publisher, New York, 1978.

[2] D. Phillips, The Technology of Fly Rods, Frank Amato Publishing Inc., Portland, Oregon, 2000. 
[3] G.A. Spolek, The Mechanics of Flycasting: Fly Line, American Journal of Physics 85 (1986), $234-240$.

[4] S. Lingard, Note on the Aerodynamics of a Flyline, American Journal of Physics 56 (1988), 756-757.

[5] J.M. Robson, The Physics of Fly Casting, American Journal of Physics 58 (1990), 234-240.

[6] C. Gatti, Numerical Simulations of Large Deformation Cable Dynamics, PhD Thesis, University of Michigan, Ann Arbor, 2002.

[7] C. Gatti and N.C. Perkins, Physical and Numerical Modeling of the Dynamics Behavior of a Fly Line, Journal of Sound and Vibration 255 (2002), 555-577.

[8] H. Ohnishi and A. Matsuzaki, The Large Deformation Analysis of a Graphite Fishing Rod, Computers and Structures 21 (1985), $265-271$.

[9] ADINA System Theory and Model Guide, section 4.3, Nonlinear Static Analysis, ADINA Engineering Inc., 1983.

[10] M.A. Hendry and M. Hubbard, Dynamic Finite Element Simulation of Fly Casting and Its Potential Use in Fly Rod Design, in: The Engineering of Sports, research, Development and Innovation, A.J. Subic and S.J. Haake, ed., Blackwell Science Ltd, Oxford, 2000, pp. 407-414.

[11] M.A. Hendry, Measurement and Simulation of Fly Casting Dynamics, MS Thesis, University of California, Davis, 1999.

[12] L. Vu-Quoc and H. Deng, GAlerkin Projection for Geometrically-exact Sandwich Beams Allowing for Ply Drop-off, ASME Journal of Applied Mechanics 62 (1995), 479-488.

[13] L. Vu-Quac and I.K. Ebcioglu, Dynamic Formulation for Geometrically-exact Sandwich Beams and 1D plates, ASME Journal of Applied Mechanics 62 (1995), 756-763.

[14] J.C. Simo and L. Vu-Quoc, A Gemometrically-exact Beam Model Incorprating Shear and Torsion Warping Deformation, International Journal of Solids and Structures 27(3) (1991), 371-393.

[15] L. Vu-Quoc and P. Legar, Efficient Evaluation of Flexibility Matrices of Tapered I-Beams Accounting for Shear Deformation, International Journal for Numerical Methods in Engineering 33 (1992), 554-566.

[16] J.C. Simo and L. Vu-Quoc, On the Dynamics of Flexible Beams Under Large Overall Motions-The Plane Case: Part I and II, ASME Journal of Applied Mechanics 53 (1986), 849-863.

[17] L. Vu-Quoc and J.C. Simo, On the Dynamics of Earth-orbiting Flexible Satellites with Multiboday Components, AIAA Journal of Guidance, Control, and Dynamics 6(6) (1987), 549-558.

[18] T.M. Wasfy and A.K. Noor, Computational Strategies for Flexible Multibody Systems, Applied Mechanics Reviews 56(6) (2003), 553-613.

[19] E. Reissner, On a One-dimensional Finite Strain Beam: The Plane Problem, Journal of Applied Mathematics and Physics 23 (1972), 795-804.

[20] S.S. Antman, Kirchhoff's Problem for Nonlinearly Elastic Rods, Quarterly Applied Mathematics 32 (1974), $221-240$.

[21] M.A. Crisfield, Non-linear Finite Element Analysis of Solids and Structures, Vol. I, Essentials, John Wiley \& Sons, Chichester, 1991.

[22] L. Vu-Quoc and I.K. Ebcioglu, General Multilayer Geometrically-exact Beams and One-dimensional Plates with Deformable Layer Thickness, Zeitschrift fur Angewandte Mathematik und Mechanik (ZAMM) 80(2) (2000), 113-135.

[23] J.C. Simo and L. Vu-Quoc, The Role of Nonlinear Theories in Transient Dynamic Analysis of Flexible Structures, Journal of Sound and Vibration 119(3) (1987), 487-508.

[24] O.C. Zienkiewizc, The Finite Element Method, McGraw-Hill, New York, 1977.

[25] K.J. Bathe, Finite Element Procedures in Engineering Analysis, Prentice-Hall, Englewood Cliffs, New Jersey, 1982.

[26] N.M. Newmark, A Method of Computation for Structural Dyanmics, ASCE Journal of Engineering Mechanics Division (1959), 67-94.

[27] T. Belytschko and T.J.R. Hughes, Klaus-Jurgen Bathe, Computational Methods for Transient Analysis, North-Holland, Amsterdam, 1983.

[28] J. Argyris and H.P. Mlejnek, Dynamics of Structures, North-Holland, New York, 1991.

[29] H. Goldstein, Classical Mechanics, Addison-Wesley Publishing Company, Menlo Park, California, 1980.

[30] C.W. Gear, Numerical Initial Value Problems in Ordinary Differential Equations, Prentice-Hall, Englewood Cliffs, New Jersey, 1971.

[31] D.G. Fertis, Nonlinear Mechanics, CRC Press, New York, 1999.

[32] P. Frank Pai, Highly Flexible Structures: Modeling, Computation, and Experimentation,AIAA Education Series, Reston, VA, 2007. 

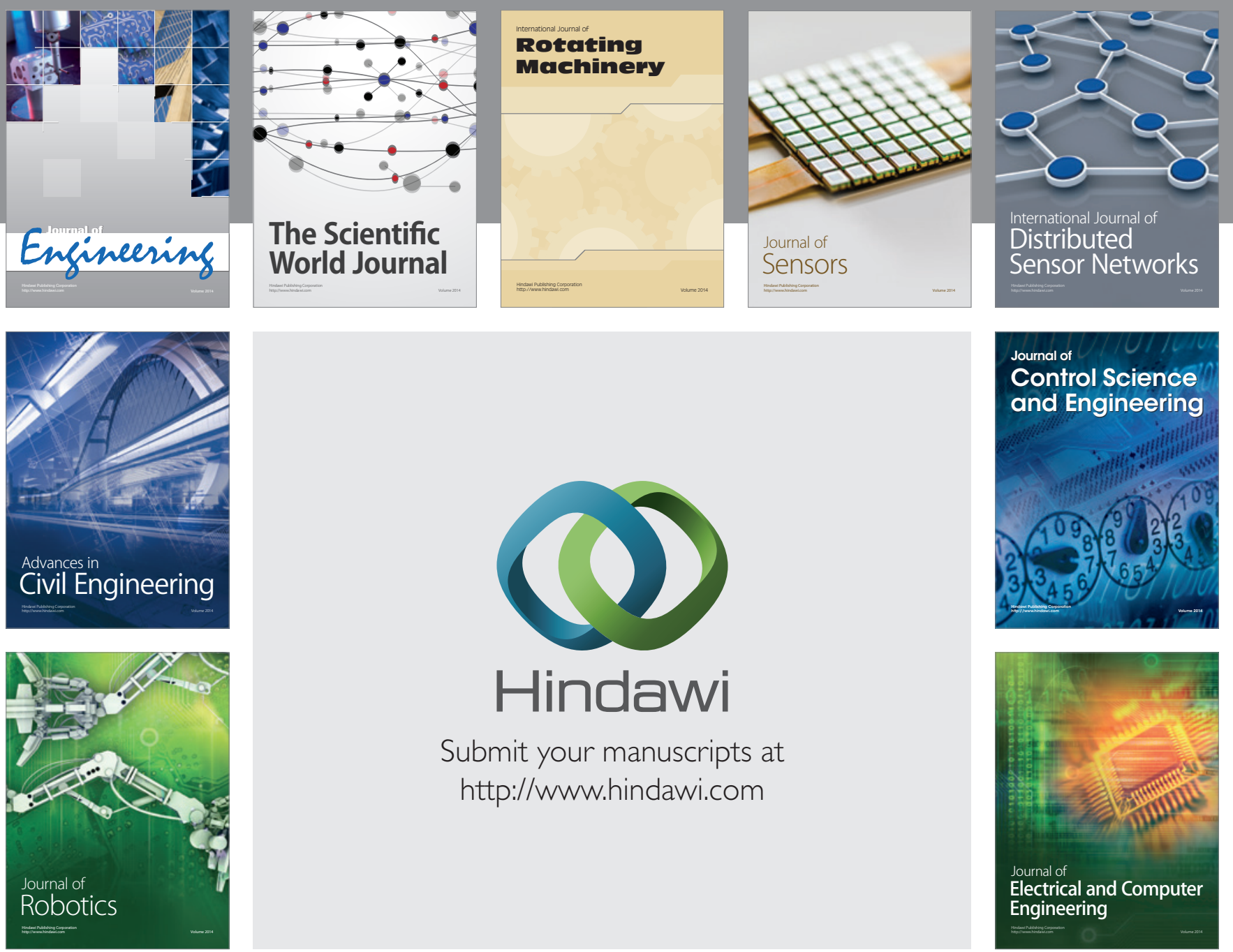

Submit your manuscripts at

http://www.hindawi.com
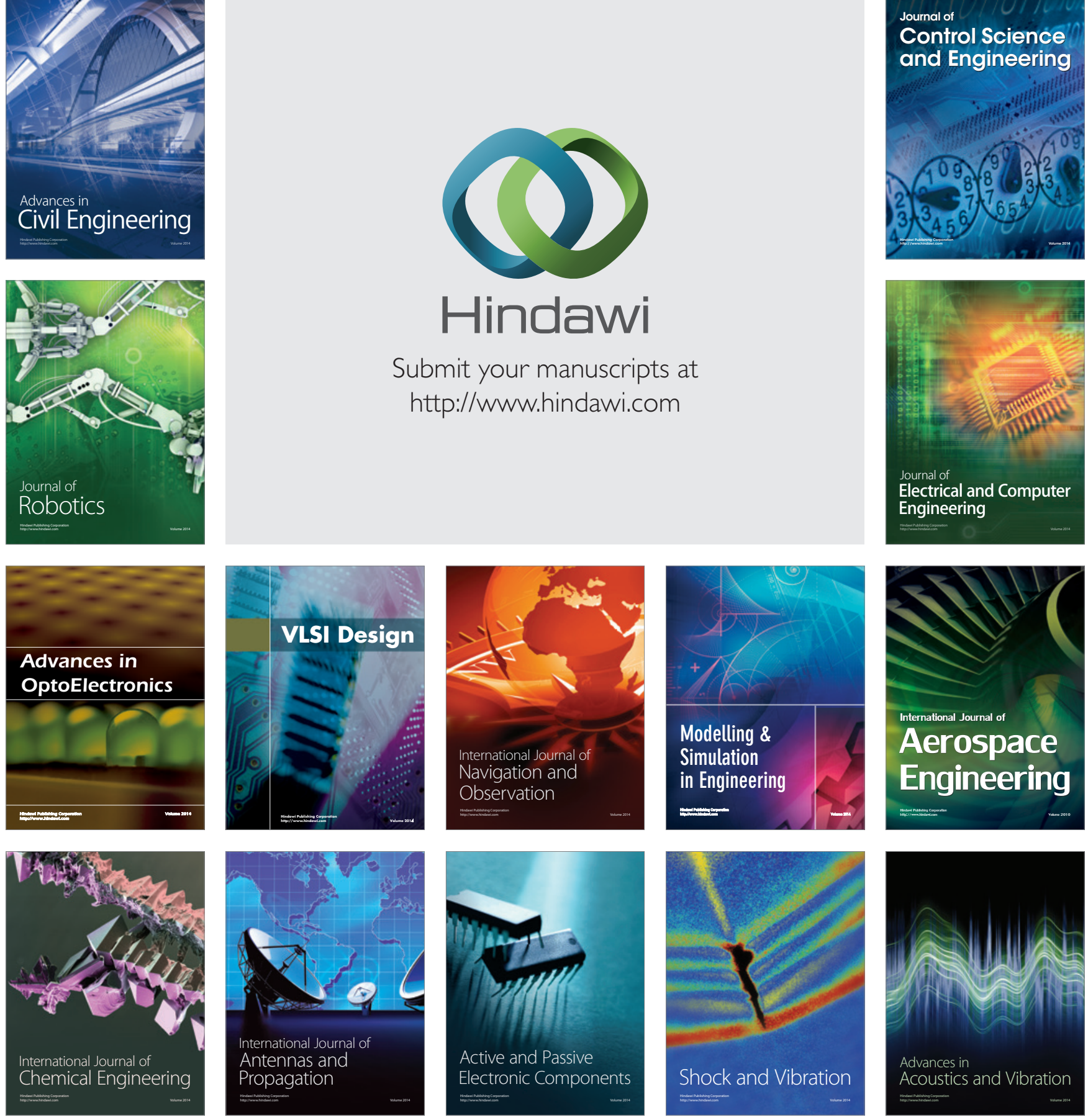